\title{
TRANSPORT COEFFICIENTS IN COLOR SUPERCONDUCTING QUARK MATTER
}

\author{
CRISTINA MANUEL* \\ Instituto de Física Corpuscular \\ Universitat de València -C.S.I.C. \\ Edificio Institutos de Paterna, \\ Apt. 2085, 46071 València. Spain \\ E-mail:Cristina.Manuel@ific.uv.es
}

\begin{abstract}
Transport coefficients of dense quark matter are needed to study properties of compact stars. They can tell us about the cooling, vibrational and rotational properties of the star. We report below a computation of the shear viscosity in the CFL phase at low temperature $T$. CFL quark matter behaves as a superfluid, and at low $T$ its transport properties are dominated by the collisions of superfluid phonons.
\end{abstract}

\section{Introduction}

The microscopic understanding of electromagnetic superconductivity is based on the seminal works of Bardeen, Cooper and Schrieffer. The BCS theory explains the main phenomenological properties of the superconductors, such as the Meissner effect, and the appearance of an energy gap $\Delta$ in the quasiparticle spectrum. It also explains the fact that superconductors are both good heat conductors and superfluids, with exponentially suppressed transport coefficients $\sim \exp [-\Delta / T]$ for low temperatures $T \ll \Delta$.

At very high density QCD is in a color superconducting phase ${ }^{1,2}$. Our present microscopic knowledge of this phenomena relies on the BCS techniques adapted to dense quark matter. From first principles one can evaluate the values of the fermionic gaps and gluon masses. There are some macroscopic properties of dense quark matter that have not been yet extensively studied. Should we expect the transport coefficients in a color superconductor to behave as in an electromagnetic superconductor? Naively, one would say so, but the answer is slightly more elaborated. Quarks have

*Work supported by MCYT (Spain) under grant FPA 2001-3031. 
different quantum numbers (spin, color, flavor), and the way they condense depends on several parameters, such as the quark masses and chemical potentials. Then one talks about different superconducting phases (CFL, 2SC, crystalline phases, gapless phases, etc), which differ basically on the way the quarks pair, and on the local or global symmetries that are spontaneously broken. Because the hydrodynamic regime of a system depends on the low energy spectrum of the theory, we could simply expect that transport coefficients of the different superconducting phases to behave very differently.

Let us insist on the relevance of transport phenomena in astrophysics ${ }^{3}$. While with the equation of state one can determine the mass and radius of a star, with the transport coefficients one can study its cooling, and its vibrational and rotational properties. Thus, they are essential for detecting signatures of quark matter in compact stars in any of its possible phases.

It has been established that the viscosities put stringent tests to astrophysical models for very rapidly rotating stars, such as for millisecond pulsars. This is based on the existence of $r$ (otational)-mode instabilities in all relativistic rotating stars ${ }^{4}$, which are only suppressed by sufficiently large viscosities. Madsen actually ruled out ${ }^{5}$ quark matter in both the CFL and 2SC phases in millisecond pulsars, because of their low viscosities. This last conclusion, however, was based on a wrong estimate, as it was assumed a behavior of the viscosities as in an electromagnetic superconductor $\sim \exp [-\Delta / T]$, for values of the temperature $T$ of the order of $0.1 \mathrm{MeV}$ and below. These estimates are not correct. In collaboration with Antonio Dobado and Felipe Llanes-Estrada we have computed the shear viscosity in the low $T$ regime of the CFL phase ${ }^{6}$ that I report below. A previous effort of studying transport properties in the 2SC phase was done in ${ }^{7}$.

\section{Transport in the CFL phase}

Transport properties in the CFL phase of QCD at low $T$ are not dominated by the quarks, which certainly give a contribution of the sort $\sim \exp [-\Delta / T]$. In this phase the diquark condensate breaks spontaneously the baryon symmetry $U_{B}(1)$, and CFL quark matter is a superfluid. Chiral symmetry is also spontaneously broken. Associated to those breakings, there are Goldstone bosons, which are light degrees of freedom. In addition, there is an unbroken $U(1)$ subgroup whose gauge boson, a combination of the photon

and one gluon, is massless at zero temperature and can be viewed as the 
in-medium photon. A CFL quark star is electrically neutral, both for the real and in-medium electromagnetism, but at finite temperature one may also expect to find electrons. All the above mentioned particles are light and their contribution to the transport coefficients in this phase is bigger than that of the gapped quarks. Let us mention that we are considering a CFL quark star after the deleptonization era, so that all the neutrinos have escaped from the star.

Chiral symmetry is not an exact symmetry of QCD. Therefore, the associated (pseudo) Goldstone bosons are massive. Their masses are estimated to be in the range of the tens of $\mathrm{MeV}$. At sufficiently low temperatures, their contribution to the viscosities is Boltzmann suppressed, and transport is dominated by the lightest particles. In particular, the highest contribution $^{8}$ is given by the Goldstone boson associated to baryon symmetry breaking, the superfluid phonon, which remains massless. There is also a contribution of the in-medium electromagnetism, but this turns out to be negligible.

The low energy effective field theory for the superfluid phonon has been constructed by Son ${ }^{9}$. He realized that the different coefficients of the corresponding effective Lagrangian can be fixed with the knowledge of the equation of state of dense quark matter. At high density, the effective Lagrangian reads

$$
L_{\mathrm{eff}}=\frac{3}{4 \pi^{2}}\left[\left(\partial_{0} \varphi-\mu\right)^{2}-\left(\partial_{i} \varphi\right)^{2}\right]^{2} .
$$

The equations of motion associated to $\varphi$ can be interpreted as hydrodynamic equations for a relativistic superfluid ${ }^{9}$, where the velocity is given by the gradient of the phase of the condensate, as in Landau's two fluid model of superfluidity. At finite $T$ they need modifications, as the different collective modes are thermally excited, and they conform a different - and dissipative - fluid. At very low $T$, dissipative effects are dominated by the collisions of the superfluid phonons.

Superfluidity in $\mathrm{He}^{4}$ is understood as a consequence of Bose-Einstein condensation. There is also a spontaneous breaking of a global $U(1)$ symmetry, with the corresponding appearance of a massless collective mode (or Goldstone boson, in the high energy language). The phonon moves at the speed of sound $v$ of the system. While in principle $\mathrm{He}^{4}$ and the CFL matter have little in common, the physics of the collective mode or phonon in the two cases exhibits many similarities, and one can then talk about universality. In particular, we found the same momentum and $T$ dependence for their damping rates. Also the mean free path has the same $T$ dependence. 
Universality aspects of these two systems are under investigation.

The physics associated to the superfluid phonon is peculiar. Even if at finite $T$ almost all particles attain a thermal mass, this is not so for the phonon, as thermal effects do not represent a violation of the $U_{B}(1)$ symmetry. The superfluid phonon suffers Landau damping, which for $p_{0}, p \ll T$ reads

$$
\operatorname{Im} \Pi\left(p_{0}, \mathbf{p}\right) \approx \frac{8 \pi^{5}}{1215} \frac{T^{4}}{v^{8} \mu^{4}} \frac{p_{0}^{3}}{p} \Theta\left(v^{2} p^{2}-p_{0}^{2}\right) .
$$

Evaluated on-shell, it gives account of its finite lifetime in the thermal bath.

Shear viscosity describes the relaxation of the momentum components perpendicular to the direction of transport, and it is usually dominated by large-angle collisions. In the CFL fluid it is not the case. The reason is that the differential cross section of binary collisions mediated by phonon exchange is divergent for small-angle collisions. It is only regulated by the finite width of the phonon, which effectively amounts to a resummation of a certain class of diagrams in the computation. Ultimately, it turns out that it is more efficient to achieve a large-angle collision by the addition of many small-angle scatterings.

With Son's effective field theory, it is possible to compute the mean free path associated to both large-angle and small-angle binary collisions. The first one behaves ${ }^{8}$ as $\sim \mu^{8} / T^{9}$ and for the values corresponding to a cold compact star, it exceeds the typical radius of a star $R \sim 10 \mathrm{~km}$, thus being irrelevant for transport. We have computed numerically the mean free path for the second ones, which parametrically behave as $\sim \mu^{4} / T^{5}$. It also exceeds the radius of a star for $T \ll 0.06 \mathrm{MeV}$, the value at which the CFL fluid in the star would behave as a perfect superfluid.

In Ref. ${ }^{6}$ we have computed numerically the shear viscosity in the CFL phase within kinetic theory, taking into account the dominant processes in the collision term. The result is consistent with a parametric behavior of the sort $\eta \sim \mu^{4} / T$. This means that the number of collisions necessary to make a large-angle collision from many small-angle ones do not depend parametrically on $T$, as one could naively expect. This is also what happens for superfluid $\mathrm{He}^{4}$, see Ref. ${ }^{10}$.

Our result could be improved by taking into account two different corrections. Namely, a better determination of the phonon interactions (determined with a more accurate equation of state of CFL quark matter), and the inclusion of the LPM effect in the collision term. We do not expect, however, that neither of these two corrections would change drastically the final result of the shear viscosity. 


\section{Conclusions}

Viscosities of the different color superconducting phases are needed for an analysis of r-mode instabillities of compact stars, which tell us about the maximum stable frequency of the star. We have focused our efforts to study the shear viscosity of the CFL phase, while the bulk viscosities are also needed and will be the subject of a different project. Our results indicate that for $T \ll 0.06 \mathrm{MeV}$ the viscosity in the phonon fluid is absent and it cannot damp the r-modes of millisecond pulsars. However, other sources of dissipation should be considered as well. In particular, in a rotating superfluid there are vortices. Particles scattering off vortices might contribute substantially to the viscosity of the rotating star. An analysis of the friction of the superfluid and the surrounding crust might also be relevant for the study of r-modes. So it is premature to discard the CFL quark matter in a millisecond pulsar, and more work is needed to draw firm conclusions about this point. Obviously, it would be equally important to evaluate the viscosities of other color superconducting phases.

\section{References}

1. K. Rajagopal and F. Wilczek, "The condensed matter physics of QCD," hep$\mathrm{ph} / 0011333$.

2. M. Alford, K. Rajagopal and F. Wilczek, Nucl. Phys. B537, 443 (1999).

3. F. Weber, "Strange quark matter and compact stars," arXiv:astro$\mathrm{ph} / 0407155$.

4. N. Andersson, Astrophys. J. 502, 708 (1998).

5. J. Madsen, Phys. Rev. Lett. 85, 10 (2000).

6. C. Manuel, A. Dobado and F. J. Llanes-Estrada, "Shear viscosity in a CFL quark star," arXiv:hep-ph/0406058.

7. D. F. Litim and C. Manuel, Phys. Rev. Lett. 87, 052002 (2001).

8. I. A. Shovkovy and P. J. Ellis, Phys. Rev. C66, 015802 (2002).

9. D. T. Son, "Low-energy quantum effective action for relativistic superfluids," arXiv:hep-ph/0204199.

10. H. J. Maris, Phys. Rev. A 8, 1980 (1973). 